\title{
Pediatric malignancies: Is the prechemotherapy left ventricular function normal?
}

\author{
Jyothsna Akam-Venkata MBBS (D) | Gilda Kadiu RDCS ｜ James Galas MD | \\ Sanjeev Aggarwal MD
}

Division of Cardiology, Carman and Ann Adams Department of Pediatrics, Wayne State University School of Medicine, Children's Hospital of Michigan, Detroit, Michigan

\section{Correspondence}

Jyothsna Akam-Venkata, MBBS, Pediatric Cardiology Fellow, The Carman and Ann Adams Department of Pediatrics, Wayne State University School of Medicine, Children's Hospital of Michigan, 3901 Beaubien Boulevard, Detroit, Michigan 48201.

Email: jyothsnaav@gmail.com

\begin{abstract}
Purpose: We compared the left ventricular (LV) systolic function in children with cancer before initiation of chemotherapy with matched controls using speckle tracking echocardiography.
\end{abstract}

Methods and results: In this retrospective study, we analyzed the echocardiograms of 89 cancer patients before the initiation of chemotherapy and 82 age- ( $8.4 \pm 5.2 \mathrm{vs}$. $8.9 \pm 3.9$ years, $P=.4$ ) and gender-matched ( $64 \%$ vs. $67 \%$, males, $P=.4$ ) healthy controls. Peak systolic LV longitudinal strain (LS) was significantly lower in cancer patients in apical two $(-19.8 \pm 3.0$ vs. $-23.5 \pm 4.0, P<.001)$, three $(-19.4 \pm 3.2$ vs. $-23.4 \pm 4.0$, $P<.001)$, and four-chamber views $(-19.7 \pm 3.4$ vs. $-22.5 \pm 3.0, P<.001)$ compared to controls, as was global longitudinal strain (GLS) $(-19.8 \pm 2.7$ vs. $-23.4 \pm 3.2, P<.001)$. The prechemotherapy group also had a higher E/e' ratio compared to controls at the septal $(9.3 \pm 3.9$ vs. $7.9 \pm 1.7, P=.005)$ and lateral annulus $(7.9 \pm 3.3$ vs. $5.9 \pm 1.4$, $P<.001)$ of the mitral valve. The LV ejection fraction was lower in cancer patients compared to controls ( $63.5 \pm 4.9$ vs. $66.8 \pm 4.1, P<.001)$, although still within normal limits. There were no differences in LV myocardial performance index $(0.30 \pm 0.05 \mathrm{vs}$. $0.30 \pm 0.09, P<.65)$ and shortening fraction $(35.8 \pm 5.2$ vs. $36.1 \pm 6.1, P<0.75)$ between the two groups. Subgroup analysis showed no difference in LV GLS between patients with solid tumors $(n=56)$ and blood cancers $(n=33)(G L S-19.2 \pm 2.9$ vs. $19.5 \pm 2.4, P>0.05$ ).

Conclusion: Our data demonstrating abnormalities in LV GLS in pediatric cancer patients even prior to initiation of chemotherapy are novel and perplexing. Further longitudinal follow-up is required to assess the implications of this abnormal LV function in these patients.

KEYWORDS

malignancy, pediatric echocardiography, strain

\section{1 | INTRODUCTION}

The outcomes of childhood cancer have improved over the last three decades with a significant decrease in mortality from 6.5 to 2 per 100000 patients. ${ }^{1}$ However, cardiovascular complications continue to be the major causes of morbidity and late mortality in pediatric cancer survivors. ${ }^{2}$ The etiology of cardiotoxicity in cancer survivors is multifactorial, with anthracycline exposure, other chemotherapeutic agents, radiation therapy, and comorbidities such as hypertension, diabetes mellitus, and coronary artery disease being major risk factors. ${ }^{3,4}$ Left ventricle (LV) dysfunction, ranging from asymptomatic dysfunction to symptomatic heart failure, can occur during 
chemotherapy or many years after the completion of chemotherapy. Echocardiography is the most commonly used noninvasive tool to assess LV function. LV ejection fraction (EF) and shortening fraction (SF) are currently used measures of assessment of systolic function in cancer patients before, during, and many years after completion of chemotherapy. ${ }^{5}$ Both these conventional echocardiographic parameters are limited in that they are dependent upon LV preload and afterload.

Speckle tracking echocardiography (STE) is a relatively newer technique which quantifies global and regional myocardial function by measuring the myocardial deformation in different phases of the cardiac cycle. Studies have shown that STE can detect abnormalities in LV function before changes in conventional echo parameters in various populations including cancer survivors exposed to cardiotoxic chemotherapy. ${ }^{6,7}$ STE is reproducible and has been shown to be independent of the preload, patient age, angle of interrogation, and ventricular geometry. ${ }^{8}$

A few studies in adult patients with cancer have reported the presence of LV dysfunction prior to initiation of chemotherapy. This finding is associated with a higher incidence of symptomatic heart failure and mortality following chemotherapy. ${ }^{6,9,10}$ Assuncao et al compared 76 adult patients with acute leukemia and 76 patients without cancer matched for age, gender, hypertension, and the presence of diabetes. Leukemia patients had a lower global longitudinal strain and higher LV mass and volumes compared to matched patients without cancer, even before receiving chemotherapy. ${ }^{11}$ Adult patients, however, have other risk factors for cardiovascular disease including diabetes mellitus, systemic hypertension, and coronary artery disease, which are very rare in children. The prevalence of LV systolic dysfunction in children with malignancies before the initiation of chemotherapy and its implications remain unknown. The primary aim of this study was to evaluate LV systolic function by STE in children with malignancies before starting chemotherapy, in comparison with matched controls. Our secondary aims were as follows: (a) to compare LV systolic function (EF and FS) and diastolic function (E/A ratio and tissue Doppler-derived E/e' ratio) in cancer patients before starting chemotherapy with healthy controls (b) to compare global function by myocardial performance index (MPI) in cancer patients before starting chemotherapy and healthy controls, and (c) to compare various echocardiographic parameters between groups of patients with solid and hematological malignancies.

\section{2 | METHODS}

This was a single-center, observational study conducted at the Children's Hospital of Michigan. Children ( $\leq 18$ years of age) diagnosed with a malignancy who had an echocardiogram performed before the initiation of chemotherapy between January 2014 and October 2016 were included as the "prechemotherapy group." Patients with (a) congenital heart defects (except patent foramen ovale), (b) dilated cardiomyopathy, (c) hemodynamically significant pericardial effusion, (d) metabolic disorders, and (5) patients who had poor quality images were excluded from the study. We also excluded patients in whom more than 2 segments were not traced properly $(n=4)$. Healthy normal children who presented to the cardiology clinic for evaluation of musculoskeletal chest pain, innocent murmurs, and vasovagal syncope and had normal electrocardiograms and echocardiograms served as controls. The study was approved by the Wayne State University School of Medicine and Detroit Medical Center Institutional Review Board. Chart review was performed and clinical data including, age, gender, and diagnosis of malignancies were recorded. Malignancies were subdivided into (a) solid tumors which included Wilm's tumor, lymphomas, osteosarcomas, and rhabdomyosarcoma and (b) hematological malignancies such as acute lymphoblastic leukemia and acute myeloid leukemia. Laboratory parameters including complete blood count with differential (including hemoglobin, hematocrit, platelets, and mean corpuscular volume), lactate dehydrogenase (LDH), serum uric acid, and serum ferritin were collected and compared between patients with solid and hematological malignancies.

\section{1 | Echocardiographic Data}

\subsection{1 | LV systolic function and LV mass}

The LV ejection fraction was calculated using the modified Simpson's method (biplane method of disk) from apical 4-chamber (Ch) and 2-Ch views as recommended by the American Society of Echocardiography and European Association of Echocardiography and the formula LVEF = (End-diastolic volume - End-systolic volume)/End-diastolic volume. ${ }^{12}$ The SF was calculated using LV (internal, septal, and posterior wall) diameters in end-diastole and end-systole on conventional $M$ mode echocardiography and the standard formula (LV end-diastolic dimension - LV end-systolic dimension)/LV end-diastolic dimension. ${ }^{13}$ LV mass was calculated from the $M$ mode measurements using the Devereux formula and was indexed to the subject's height $(\mathrm{cm})^{2.7} \mathrm{LV}$ mass $=0.8\{1.04[([\mathrm{LVE}$ $\left.\left.\left.\mathrm{Dd}+\mathrm{IVSd}+\mathrm{PWd}^{3}-\mathrm{LVEDd}^{3}\right)\right]\right\}+0.6$ where LVEDd, IVSd, and PWd represent $L V$, interventricular septal, and posterior wall thickness in diastole, respectively. ${ }^{14}$

\subsection{2 | $\mathrm{LV}$ diastolic function}

For assessment of diastolic function, the ratio of the velocity of early diastolic filling $(E)$ to the velocity of diastolic filling due to atrial contraction (A) was measured from the spectral Doppler of LV inflow (E/A) (Figure 1A). Tissue Doppler-derived velocities of the septal and the lateral annulus of the mitral valve due to early diastolic filling (e'), diastolic filling due to atrial contraction (a'), and velocity due to ventricular systole (s') were analyzed as well (Figure 1C). ${ }^{13}$

\subsection{3 | LV global function}

Global LV function was assessed using MPI, calculated as the ratio of the sum of the time spent in isovolumetric contraction (ICT) and 

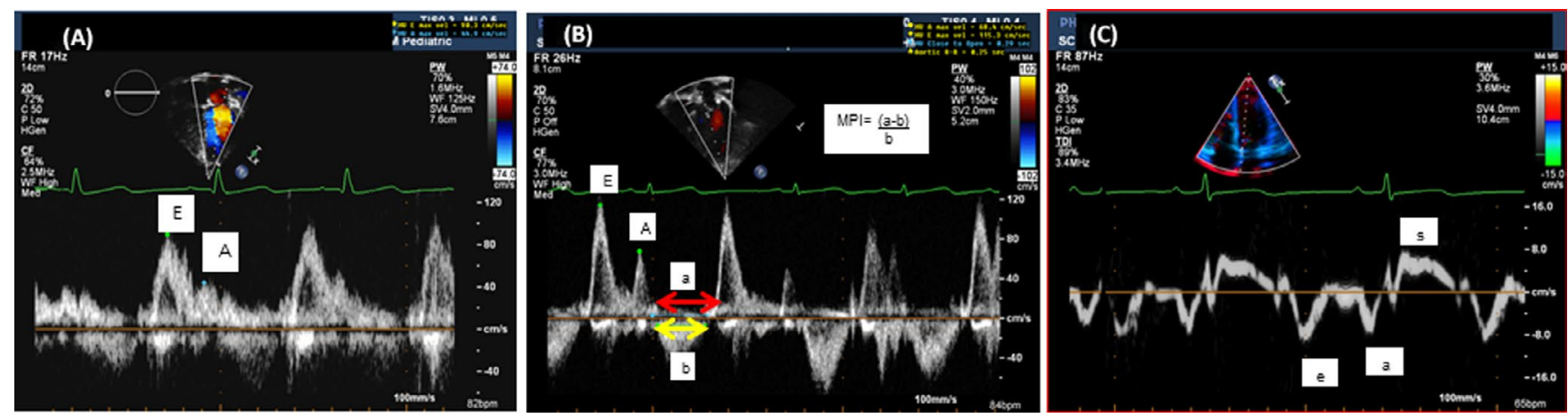

FIG URE 1 Spectral (A and B) and tissue Doppler (C) imaging of the mitral valve

FIGURE 2 Longitudinal strain with bull's eye plot measured from 4-chamber view

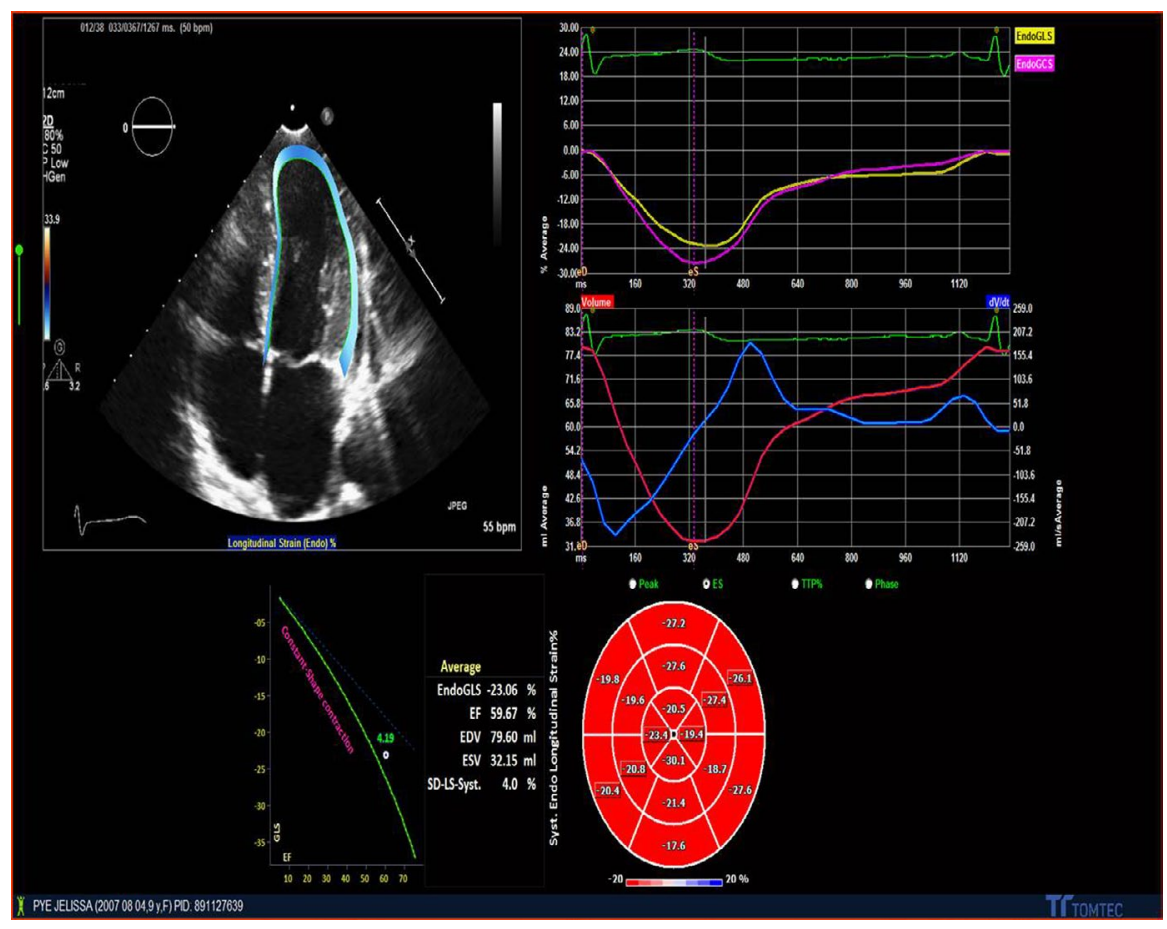

relaxation phase (IRT) divided by the ejection time (ET) as shown in Figure $1 \mathrm{~B} .^{15}$

\subsection{4 | Speckle Tracking Echocardiography}

The echocardiograms were performed using iE 33 (Phillips) and stored in compressed Digital Imaging and Communication in Medical (DICOM) format for offline analysis. Two-dimensional peak systolic longitudinal strain (LS) was measured using vendorindependent software (2D Cardiac Performance Analysis, Tom Tec Imaging Systems). The images were analyzed using the single best loop from the apical 2-Ch, 3-Ch, and 4-Ch views. The LV endocardial border was traced semi-automatically in systole. The images were played and tracings were manually adjusted when appropriate. The software measured the LS from $2-\mathrm{Ch}, 3-\mathrm{Ch}$, and $4-\mathrm{Ch}$ views and calculated the global peak systolic longitudinal strain (GLS) as an average of these three values (Figure 2). These analyses were performed by a single reader who was blinded to clinical details (GK).

\section{2 | Statistical Analysis}

Continuous variables were expressed as mean (SD), median (IQR), and categorical variables were expressed as number (\%). The two groups with and without malignancy were compared using Student's t test, Chi-square test, and Mann-Whitney $U$ test as appropriate. Subgroup analyses included a comparison of biochemical markers and echocardiographic parameters in the groups with solid and hematologic malignancies using nonparametric MannWhitney $\mathrm{U}$ test. Linear regression analysis was performed to identify risk factors associated with abnormal GLS, adjusting for age and gender. The intra-class correlation coefficient (ICC) was calculated to assess the inter-observer variability in a random sample of 20 patients. Statistical analyses were performed using SPSS 
TAB LE 1 Demographic data in prechemotherapy group and healthy controls

\begin{tabular}{|llcl|}
\hline & $\begin{array}{l}\text { Prechemotherapy } \\
\text { group } \\
\text { (n= 89) }\end{array}$ & $\begin{array}{l}\text { Healthy controls } \\
(\mathbf{n = 8 2})\end{array}$ & $\mathbf{P}$ \\
\hline Age (y) & $8.4(5.2)$ & $8.9(3.9)$ & .4 \\
\hline Gender (male) & $57(64 \%)$ & $55(67 \%)$ & .4 \\
\hline Weight (kg) & $34.1(24.3)$ & $36.8(19.3)$ & .43 \\
\hline Height (cm) & $125(33)$ & $134.3(27.1)$ & $.046^{*}$ \\
\hline $\begin{array}{c}\text { Systolic BP } \\
\text { (mm Hg) }\end{array}$ & $108(15)$ & $107(13)$ & .8 \\
\hline $\begin{array}{c}\text { Diastolic BP } \\
(\mathrm{mm} \mathrm{Hg})\end{array}$ & $64(12)$ & $61(8)$ & .08 \\
\hline
\end{tabular}

${ }^{*} P<.05$

version 21 (SPSS, Chicago, IL, USA), and significance was defined by $p$-value $<0.05$.

\section{3 | RESULTS}

Our cohort consisted of a total of 171 patients, of whom 89 (52\%) were cancer patients who had an echocardiogram performed before initiation of chemotherapy, and 82 (48\%) were healthy controls. The two groups were comparable in age (Mean (SD) $8.4 \pm 5.2$ vs. $8.9 \pm 3.9$ years, $P=.4$ ), weight ( $34.1 \pm 24.3$ vs. $36.8 \pm 19.3 \mathrm{Kg}, P=.43$ ) and gender distribution ( $64 \%$ vs. $67 \%$ males, $P=.40$ ) as shown in Table 1. Among the 89 patients in the prechemotherapy group, 56 (62.9\%) had solid tumors and 33 (37.1\%) had hematological tumors; the different sub-types of tumors are summarized in Table 2.

\section{1 | Prechemotherapy group vs. healthy controls}

\subsection{1 | Conventional echocardiography}

The pediatric cancer patients had a lower LV EF compared to the matched healthy controls ( $63.5 \pm 4.9$ vs. $66.8 \pm 4.1, P<.001)$, although still within normal limits. The SF $(35.8 \pm 5.2$ vs. $36.1 \pm 6.1$, $P=.75)$, LV mass indexed to $\mathrm{Ht}^{2.7}(36.9 \pm 19.2$ vs. $32.5 \pm 9.1$, $P=.06)$, and MPI, a marker of global LV function (0.30 \pm 0.05 vs. $0.30 \pm 0.09, P=.65)$, were not significantly different between groups. The prechemotherapy group of patients had a smaller LV internal diameter in diastole ( $3.9 \pm 0.8$ vs. $4.15 \pm 0.7, P=.02)$, higher $E / e^{\prime}$ ratio at the septal $(9.3 \pm 3.9$ vs. $7.9 \pm 1.7, P=.005)$ and lateral annulus $(7.9 \pm 3.3$ vs. $5.9 \pm 1.4, P<.001)$ of the mitral valve and lower E/A ratio (1.6 \pm 0.48 vs. $2.3 \pm 0.72, P<.001)$, compared to healthy controls (Table 3 ).

\subsection{2 | Speckle tracking echocardiography}

The LS was significantly lower in the prechemotherapy group compared to healthy controls in the 2 -Ch $(-19.8 \pm 3.0$ vs. $-23.5 \pm 4.0$, $P<.001)$, 3 -Ch (-19.4 \pm 3.2 vs. $-23.4 \pm 4.0, P<.001)$, and $4-C h$ views
TAB LE 2 Distribution of different types of solid and hematological tumors

\begin{tabular}{|l|l|}
\hline Type of malignancy & $\mathrm{n}(\%)$ \\
\hline Solid tumors $(\mathrm{n}=56,62.9 \%)$ & \\
\hline Neuroblastoma & $9(10.1)$ \\
\hline Osteosarcoma & $8(9.0)$ \\
\hline Hodgkin's lymphoma & $8(9.0)$ \\
\hline Wilm's tumor & $7(7.9)$ \\
\hline T-cell Lymphoblastic lymphoma & $6(6.7)$ \\
\hline Rhabdomyosarcoma & $4(4.5)$ \\
\hline Ewing sarcoma & $3(3.4)$ \\
\hline Burkitt lymphoma & $3(3.4)$ \\
\hline Embryonal sarcoma & $2(2.2)$ \\
\hline Hepatoblastoma & $2(2.2)$ \\
\hline Ependymoma & $1(1.1)$ \\
\hline Small round cell tumor & $1(1.1)$ \\
\hline Hepatocellular carcinoma & $1(1.1)$ \\
\hline Basal cell carcinoma & $1(1.1)$ \\
\hline Hematological tumors ( $=33,37.1 \%)$ & \\
\hline B precursor-Acute Lymphoblastic leukemia & $22(24.7)$ \\
\hline T precursor-Acute Lymphoblastic leukemia & $4(4.5)$ \\
\hline Acute myeloblastic leukemia & $6(6.7)$ \\
\hline Acute biphenotypic (lymphoid + myeloid) leukemia & $1(1.1)$ \\
\hline
\end{tabular}

$(-19.7 \pm 3.4$ vs. $-22.5 \pm 3.0, P<.001)$ as shown in Table 3 and Figure 3. Also, GLS was significantly lower in the prechemotherapy group compared to the healthy controls $(-19.8 \pm 2.9$ vs. $-23.4 \pm 3.2, P<.001)$. A total of $26(29 \%)$ cancer patients had a GLS lower than the 5 th percentile $(-18.75)$ of healthy controls. On regression analysis, we found that a diagnosis of cancer was independently associated with abnormalities in GLS, after adjusting for age and gender (Table 4).

\subsection{3 | Inter-observer Variability}

The intra-class correlation coefficient (ICC) for LS was $0.94(95 \%$ confidence interval $0.86-0.98, f$ value $17.16, P<.001)$ indicating minimal inter-observer variability.

\subsection{Solid vs. Hematological malignancies}

There were no differences in age, weight, height, heart rate, and blood pressure between patients with solid $(n=56)$ and hematological malignancies $(n=33)$ (Table 5). Patients with hematological tumors had a higher white count, were more anemic, and had significantly higher $\operatorname{LDH}(P=.003)$, uric acid $(P<.001)$, and ferritin $(P<.001)$ compared to those with solid tumors (Table 6).

\subsection{1 | Conventional echocardiography}

Spectral and tissue Doppler-derived measurements of diastolic function such as E/A ratio, E/e' ratio at the septal and the lateral 
TABLE 3 Echocardiographic parameters in prechemotherapy group and healthy controls

\begin{tabular}{|lccc|}
\hline Mean (SD) & $\begin{array}{l}\text { Prechemotherapy group } \\
(\mathbf{n}=89)\end{array}$ & $\begin{array}{l}\text { Controls } \\
(\mathbf{n}=82)\end{array}$ & $P$ \\
\hline LV 2-Ch LS & $-19.8(3.0)$ & $-23.5(4.0)$ & $<.001^{*}$ \\
\hline LV 3-Ch LS & $-19.4(3.2)$ & $-23.4(4.0)$ & $<.001^{*}$ \\
\hline LV 4-Ch LS & $-19.7(3.4)$ & $-22.5(3.0)$ & $<.001^{*}$ \\
\hline LV GLS & $-19.8(2.7)$ & $-23.4(3.2)$ & $<.001^{*}$ \\
\hline LV EF \% & $63.5(4.9)$ & $66.8(4.1)$ & $<.001^{*}$ \\
\hline LV FS \% & $35.8(5.2)$ & $37.7(11.2)$ & .15 \\
\hline LVIDD (cm) & $3.9(0.8)$ & $4.15(0.7)$ & $.02^{*}$ \\
\hline LV mass indexed to Ht2.7 & $36.9(19.2)$ & $32.5(9.1)$ & .06 \\
\hline E/A ratio & $1.6(0.48)$ & $2.3(0.72)$ & $.001^{*}$ \\
\hline E/e' septal & $9.3(3.9)$ & $7.9(1.7)$ & $.005^{*}$ \\
\hline E/e' lateral & $7.9(3.3)$ & $5.9(1.4)$ & $<.001^{*}$ \\
\hline MPI & $0.30(0.05)$ & $0.30(0.09)$ & .65 \\
\hline
\end{tabular}

Abbreviations: LV, left ventricle; Ch, chamber; LS, peak systolic longitudinal strain; GLS, average peak systolic longitudinal strain; EF, ejection fraction; FS, fractional shortening; LVIDD, left ventricular internal diameter in diastole; $E$, velocity of early diastolic filling of the left ventricle; $A$, velocity of diastolic filling of the left ventricle due to atrial contraction; e', tissue Doppler-derived velocity of the septal and the lateral annulus of the mitral valve due to early diastolic filling; MPI, myocardial performance index.

${ }^{*} P<.05$ annulus of the mitral valve, and LV systolic function measured by EF and SF were not significantly different among patients with solid and hematological tumors. Even though MPI was statistically different between the two subgroups, it was in the normal range for both groups (Table 5).

\subsection{2 | Speckle tracking echocardiography}

LV LS measured from 2-Ch, 3-Ch, and 4-Ch views and GLS were not different between these groups (Table 5).

\section{4 | DISCUSSION}

The present study demonstrates that children with cancers have abnormal LV systolic function, reflected by decrease in peak systolic longitudinal strain (LS) in different planes (2-Ch, 3-Ch, and 4-Ch apical views) as well as a decrease in global longitudinal strain (GLS) prior to exposure to chemotherapy when compared to matched healthy controls. The diagnosis of cancer was independently associated with abnormalities in GLS, after adjusting for the patient's age and gender. Interestingly, all the patients had normal conventional parameters such as SF and MPI. Our findings are similar to recently published results in adults (median age: 56 years) by Assuncao et al of lower GLS $(-19.3 \pm 2.7$ vs. $20.9 \pm 1.9, P<.001)$ but similar LV EF ( $62 \pm 6$ vs. $62 \pm 5, P=.34)$ in the group with acute leukemia compared to matched patients without cancer. ${ }^{11}$

In adults with cancers, a baseline abnormal strain prior to initiating chemotherapy has been shown to be associated with adverse cardiovascular risk following chemotherapy. ${ }^{9,16}$ Ali et al followed 450 adult leukemia patients for a median duration of 159 days (range 13-2891 days) after the start of chemotherapy and found that 28 (6\%) patients had cardiac events (defined as death or symptomatic heart failure with a decrease in EF (58 \pm 10 vs. $62 \pm 7, P=.005)$ ). Patients with cardiac events had significantly lower prechemotherapy GLS $(-15 \pm 2.8 \%$ vs. $-19.7 \pm 2.7, P<.001)$. Interestingly, absolute GLS $<-17.5 \%$ was associated with a sixfold increase in cardiac events. ${ }^{9}$ Among 2234 adult patients with breast and hematological cancers, 158 (7\%) had LVEF of 50\%-59\% before initiation of chemotherapy. ${ }^{16}$ Over a median duration of 659 days, 12 (7.6\%) patients in this group developed major adverse cardiac events (heart failure or death) at a median duration of 173 days. Baseline GLS was significantly lower (-16 \pm 2.5 vs. $-17.7 \pm 2.6, P=.015)$ compared to patients without events and independently predicted a major adverse cardiovascular event defined as New York Heart Association class III, IV congestive heart failure or cardiac death $(P=.0065) .{ }^{16}$ In a prospective study of 86 adults with various malignancies receiving anthracyclines (median age 48, range 30-63.5 years), 6 patients developed a decrease in LV EF by more than $10 \%$ to below $53 \%$ one year after completing chemotherapy. GLS measured before initiation of chemotherapy was significantly lower (-19.1 vs. $-21.2, P=.042)$ in the group that developed anthracycline-induced cardiotoxicity compared to the group that did not. ${ }^{17}$ Abnormal LV systolic function, defined by LVEF less than $55 \%$, was noted in 12 of 88 (13\%) patients with breast cancer and leukemia even before the initiation of chemotherapy. These patients had higher mortality and comprised $40 \%$ (4 out of 10 total deaths) of the mortality after chemotherapy compared to patients with normal LV function before chemotherapy. ${ }^{18}$ 

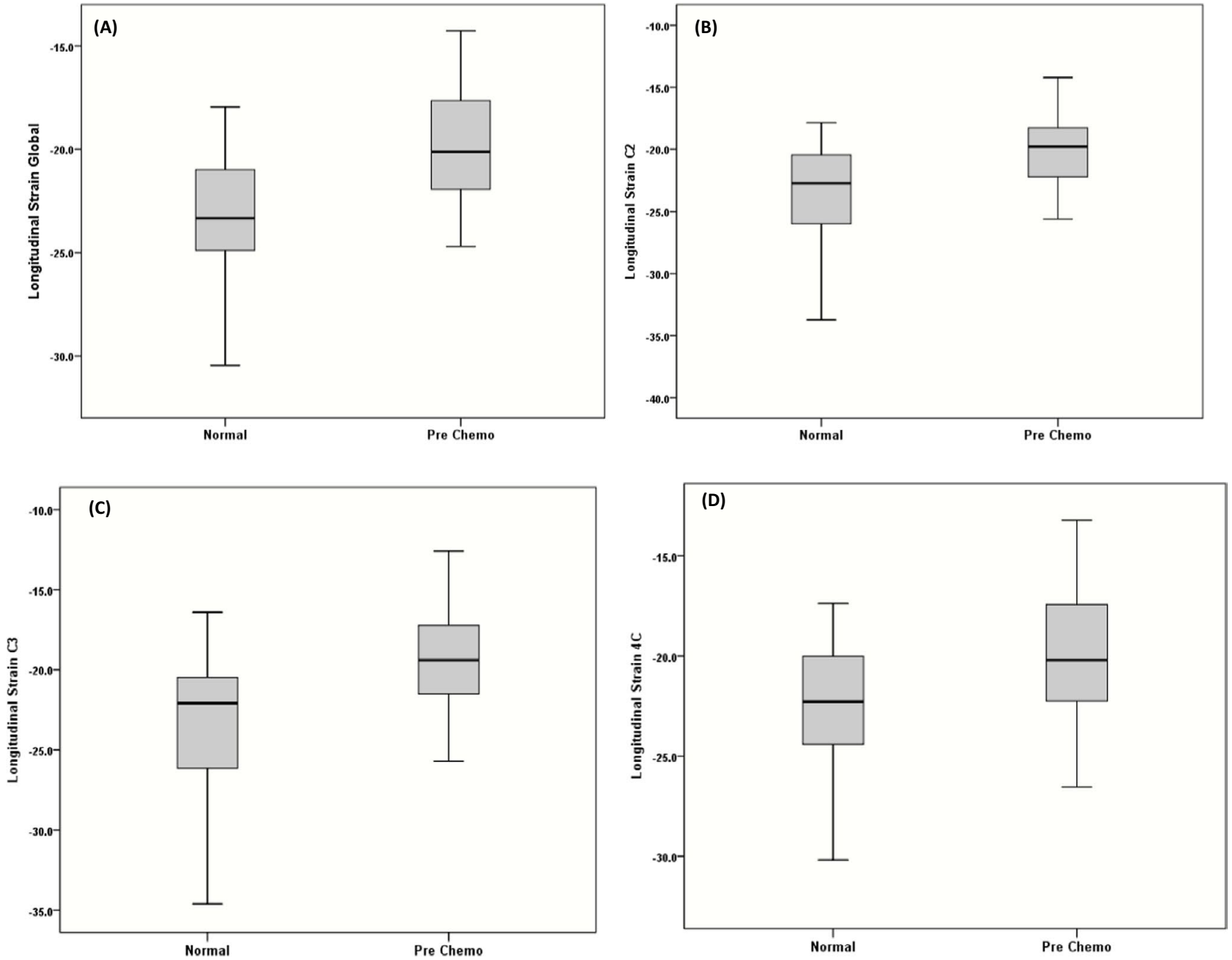

FIGURE 3 Box and Whisker plot comparing global longitudinal strain (A), longitudinal strain in 2-chamber (B), 3-chamber (C), and 4chamber views (D) between healthy controls and cancer patients

Changes in the LV GLS from baseline have been noted in early phases of chemotherapy and also have been shown to be associated with subsequent LV dysfunction during follow-up. Asymptomatic patients $(\mathrm{n}=19)$ who received anthracycline (mean dose, $296 \pm 103 \mathrm{mg} / \mathrm{m}^{2}$ ) therapy showed a significant decrease in LV GLS at 4 (change $8.7 \% \pm 0.2 \%, P=.033$ ) and 8 months (change $9.2 \pm 0.3 \%, P=.015)$ from their baseline value $(-19.9 \pm 2.1)$ and compared to age- and gender-matched controls $(20.5 \pm 1.5 \%$, $P=.011)$. LV EF decreased significantly at 8 months $(4.3 \pm 0.1 \%$, $P=.044)$ and correlated with the segmental changes in the mid and apical LV longitudinal peak systolic strain. ${ }^{7}$ Among 43 breast cancer patients, 8 developed cardiotoxicity at 6 months following the start of chemotherapy, defined as more than $5 \%$ decrease in LV EF to $<55 \%$ in symptomatic heart failure or more than $10 \%$ decrease in asymptomatic patients. A decrease in longitudinal $(-20.5 \pm 2.2$ to $-19.3 \pm 2.4, P=.01)$ and radial $(55 \pm 12$ to $52 \pm 12$, $P=.02)$ strain, from baseline to 3 months independently predicted cardiotoxicity at 6 months. ${ }^{6}$
TAB LE 4 Regression analysis showing association between diagnosis of cancer and global longitudinal strain

\begin{tabular}{llll}
$\begin{array}{l}\text { Independent } \\
\text { Variable }\end{array}$ & $\boldsymbol{\beta}$ coefficient & $\begin{array}{l}\text { 95\% confidence } \\
\text { interval }\end{array}$ & $\boldsymbol{P}$ \\
\hline $\begin{array}{l}\text { Global longitudi- } \\
\text { nal strain }\end{array}$ & 0.42 & $1.86,3.98$ & $<.001$ \\
Age & 0.12 & $-0.02,0.21$ & .11 \\
\hline Gender & 0.03 & $-0.91,1.32$ & .73 \\
\hline
\end{tabular}

STE is becoming increasingly popular in the assessment of subclinical LV dysfunction. Abnormalities in strain are reported to occur even before changes in conventional echo parameters. A meta-analysis of one retrospective and 15 prospective studies involving 5721 patients with heart failure, myocardial infarction, valvular heart diseases, has shown that decrease in global longitudinal strain is independently associated with mortality and has superior prognostic value to LV EF. ${ }^{19}$ STE is accurate and validated as a measure of 
TAB LE 5 Comparison of echocardiographic parameters in Solid and hematological tumors

\begin{tabular}{|lccc} 
Mean (SD) & $\begin{array}{l}\text { Solid tumors } \\
(\mathbf{n}=56)\end{array}$ & $\begin{array}{l}\text { Hematological } \\
\text { tumors }(\mathbf{n}=33)\end{array}$ & $\mathbf{P}$ \\
\hline Age $(\mathrm{y})$ & $8.8(5.3)$ & $7.7(5.3)$ & .36 \\
\hline Height $(\mathrm{cm})$ & $127(32.5)$ & $121.7(34.1)$ & .47 \\
\hline Weight $(\mathrm{kg})$ & $33.8(21.8)$ & $34.5(29.3)$ & .91 \\
\hline LV 2-Ch LS & $-18.6(3.2)$ & $-19.7(3)$ & .3 \\
\hline LV 3-Ch LS & $-19.2(4)$ & $-19.1(2.9)$ & .9 \\
\hline LV 4-Ch LS & $-19.2(3.6)$ & $-18.7(4)$ & .6 \\
\hline LV GLS & $-19.2(2.9)$ & $-19.5(2.4)$ & .7 \\
\hline MPI & $0.31(0.05)$ & $0.28(0.05)$ & $.04^{*}$ \\
\hline E/A ratio & $1.69(0.5)$ & $1.63(0.4)$ & .5 \\
\hline E/e' Septal & $9.6(4.7)$ & $8.9(1.8)$ & .4 \\
\hline E/e' lateral & $7.9(3.9)$ & $7.7(1.7)$ & .7 \\
\hline Ejection & $63.6(5.1)$ & $63.2(4.5)$ & .75 \\
\hline Fraction & & $34.8(4.5)$ & .1 \\
\hline Shortening & $36.5(5)$ & & \\
\hline Fraction & & & \\
\hline
\end{tabular}

Abbreviations: LV, left ventricle; Ch, chamber; LS, peak systolic longitudinal strain; GLS, average peak systolic longitudinal strain; E, velocity of early diastolic filling of the left ventricle; A, velocity of diastolic filling of the left ventricle due to atrial contraction; e', tissue Doppler-derived velocity of the septal and the lateral annulus of the mitral valve due to early diastolic filling; MPI, myocardial performance index. ${ }^{*} P<.05$

myocardial strain. ${ }^{8,20}$ The American Society of echocardiography and European Association of Cardiovascular imaging recommend serial measurements of global longitudinal strain, ideally beginning from prior to initiation of chemotherapy. The global longitudinal strain is an ideal deformation parameter in the early detection of subclinical LV dysfunction defined as $>15 \%$ decrease from the baseline value. ${ }^{21}$

In the current study, we found evidence of a difference in diastolic function parameters (E/e' and E/A ratio) in cancer patients and controls. Also, the $E / \mathrm{e}^{\prime}$ ratio was higher in cancer patients at the septal $(9.3 \pm 3.9$ vs. $7.2 \pm 1.6)$ and the lateral annulus $(7.9 \pm 3.3$ vs. $5.8 \pm 1.9)$ of the mitral valve, compared to previously published values in 55 healthy children between 6 and 9 years. ${ }^{22}$ Corresponding mean E/A ratios were $1.6 \pm 0.48$ in our cancer cohort, $2.3 \pm 0.72$ in the controls, and $1.99 \pm 0.51$ in the published normative study. However, the clinical significance of these differences in values is uncertain and whether they indicate subtle diastolic dysfunction cannot be established.

Diastolic dysfunction in adult cancer patients exposed to chemotherapy has been reported previously. ${ }^{23}$ A pediatric study reported normal diastolic function, as assessed by E/A ratio of $2.1 \pm 4.3$, septal $E / E^{\prime}$ ratio of $7.2 \pm 2$ and lateral $E / E^{\prime}$ ratio of $5.5 \pm 1.2$, in 63 cancer patients aged $13.7 \pm 4.5$ years, 5 years from completion of anthracycline chemotherapy with a median cumulative dose of 165 (range $45-520) \mathrm{mg} / \mathrm{m}^{2} .{ }^{24}$ In contrast, an increase in MPI with increasing doses of anthracycline exposure from 200 to $300 \mathrm{mg} / \mathrm{m}^{2}$ dose has been demonstrated. ${ }^{15}$ However, data on diastolic function or global
TAB LE 6 Biochemical and Hematological parameters in Solid and Hematological tumors

\begin{tabular}{|c|c|c|c|}
\hline Mean (SD) & $\begin{array}{l}\text { Solid tumors } \\
(n=56)\end{array}$ & $\begin{array}{l}\text { Hematological } \\
\text { tumors }(n=33)\end{array}$ & $P$ \\
\hline Hemoglobin (g/dL) & $11.4(3.6)$ & $8.4(3.3)$ & $<.001^{*}$ \\
\hline $\begin{array}{l}\text { WBC }(\times 103 \text { cells/ } \\
\text { mm3) }\end{array}$ & $10.2(9.4)$ & $57.2(82.8)$ & $<.001^{*}$ \\
\hline $\begin{array}{l}\text { Platelets ( } \times 103 \text { cells/ } \\
\text { mm3) }\end{array}$ & 304 (138) & 89 (110) & $<.001^{*}$ \\
\hline Retic count (\%) & $1.8(1.2)$ & $0.98(0.7)$ & $<.001^{*}$ \\
\hline Ferritin (ng/mL) & $51.5(31)$ & 401(309) & $<.001^{*}$ \\
\hline LDH (units/L) & $548(482)$ & $1344(1670)$ & $.002^{*}$ \\
\hline Uric acid (mg/dL) & $3.7(1.4)$ & $5.9(3)$ & $<.001^{*}$ \\
\hline
\end{tabular}

Abbreviations: LDH, lactate dehydrogenase; WBC, white blood cell count; Retic, reticulocyte, ng, nanogram; $\mathrm{mL}$, milliliter; L-liter. ${ }^{*} P<.05$

myocardial performance in pediatric cancer patients before initiating chemotherapy are lacking. In our study, MPI was similar in cancer patients and healthy controls.

\subsection{Effect of Hematological cancer vs. Solid cancers}

In our study, there were no significant differences in GLS in patients with leukemia and solid tumors. This is in contrast to the reported higher abnormalities in adults with leukemia, compared to solid tumors. In a subgroup analysis, 28 women with leukemia showed significantly lower GLS $(-19.4 \pm 3 \%$ vs. $-21.2 \pm 2.2 \%)$, compared to 28 women with breast cancer and $(-21.5 \pm 2.1 \%)$ noncancer patients, with no differences in LV volumes and mass. ${ }^{11}$ Ali et al reported a higher incidence of symptomatic heart failure and cardiac death in adults with acute leukemia (10 of $80,13 \%$ ) compared to lymphoma (18 of $370,5 \%$ ) following anthracycline treatment.

\section{2 | Plausible etiology for prechemotherapy LV dysfunction}

Several factors may explain the abnormalities in LV function before the initiation of chemotherapy. Inflammatory mediators have been postulated to play a role in LV remodeling. In animal models, changes in the expression of TNF and total matrix metalloproteinase inhibitor activities have been associated with LV dilation or remodeling. ${ }^{25}$ Alternatively, infiltration of the myocardium by circulating cancer cells could cause cardiac dysfunction. In an autopsy study of 420 hearts with leukemia, leukemic infiltrates and hemorrhage were observed in $69 \%$. LV was the second most common location of leukemic infiltrates (36\%) after pericardium (39\%). ${ }^{26}$

In this novel study of LV function in pediatric cancer patients before initiation of chemotherapy, abnormal LV systolic deformation, as evidenced by changes in strain parameters, was detected even though conventional echocardiographic systolic parameters were mostly within the normal range. We also demonstrated alteration 
in diastolic function as measured by the $\mathrm{E} / \mathrm{e}^{\prime}$ ratio in this group. The prechemotherapy global longitudinal strain may serve as an important risk stratification tool in identifying cancer patients who are at risk for subsequent chemotherapy-related cardiovascular toxicity. These patients may need closer monitoring and may benefit from prompt intervention or prophylactic treatment.

\section{3 | Limitations}

The present study is limited by its retrospective nature and singlecenter data. Even though this is retrospective data, we had sufficient quality echocardiographic images to measure the longitudinal strain in different planes from apical 2-Ch, 3-Ch, and 4-Ch images as well as global longitudinal strain. In our center, all cancer patients undergo echocardiographic evaluation before starting anthracycline-based chemotherapy. However, cancer patients not receiving anthracycline chemotherapy may have been missed. Data on markers of inflammation (cytokines, $\mathrm{C}$ reactive protein, and erythrocyte sedimentation rate), cardiac biomarkers ( $\mathrm{N}$-terminal pro-brain natriuretic peptide, cardiac troponins, and creatine kinase) were not available in cancer patients before initiation of chemotherapy. Our study was designed to evaluate the presence of abnormal GLS in the prechemotherapy setting; therefore, longitudinal follow-up to assess the prognostic implications of our findings was not performed.

\section{5 | CONCLUSION}

Our study concludes that abnormal LV systolic global longitudinal strain is present in a significant number of children with cancer prior to exposure to chemotherapeutic agents. A larger multicenter longitudinal follow-up study is needed to validate our results and assess the utility of prechemotherapy strain in identifying those at risk of post-chemotherapy-cardiotoxicity. Further investigation into the etiology and prevention of abnormal GLS even before exposure to chemotherapy in cancer patients is needed.

\section{CONFLICT OF INTEREST}

The authors declare that they have no conflict of interest.

\section{ETHICAL APPROVAL}

This article does not contain any studies with human participants or animals performed by any of the authors. The study was approved by the Wayne State University School of Medicine and Detroit Medical Center Institutional Review Board.

\section{INFORMED CONSENT}

Not applicable. Waiver of consent obtained through Wayne State University School of Medicine and Detroit Medical Center Institutional Review Board.
ORCID

Jyothsna Akam-Venkata (iD) https://orcid.

org/0000-0001-8625-6020

\section{REFERENCES}

1. AmericanCancerSociety. Cancer Facts \& Figures 2017. Available from: https://www.cancer.org/content/dam/cancer-org/research/ cancer-facts-and-statistics/annual-cancer-facts-and-figures/2017/ cancer-facts-and-figures-2017.pdf

2. Mertens AC, Liu Q, Neglia JP, et al. Cause-specific late mortality among 5-year survivors of childhood cancer: the childhood cancer survivor study. J Natl Cancer Inst. 2008;100(19):1368-1379.

3. Gilladoga AC, Manuel C, Tan C, Wollner N, Sternberg SS, Murphy ML. The cardiotoxicity of adriamycin and daunomycin in children. Cancer. 1976;37(2 Suppl):1070-1078.

4. Lipshultz SE, Adams MJ, Colan SD, et al. Long-term cardiovascular toxicity in children, adolescents, and young adults who receive cancer therapy: pathophysiology, course, monitoring, management, prevention, and research directions: a scientific statement from the American Heart Association. Circulation. 2013;128(17):1927-1995.

5. Children'sOncologyGroup. Long-Term Follow-up Guidelines for Survivors of Childhood, Adolescent and Young Adult Cancer 2013, October [Version 4.0]. Available from: http://www.survivorshipgui delines.org/pdf/LTFUGuidelines_40.pdf

6. Sawaya H, Sebag IA, Plana JC, et al. Early detection and prediction of cardiotoxicity in chemotherapy-treated patients. Am J Cardiol. 2011;107(9):1375-1380.

7. Poterucha JT, Kutty S, Lindquist RK, Li L, Eidem BW. Changes in left ventricular longitudinal strain with anthracycline chemotherapy in adolescents precede subsequent decreased left ventricular ejection fraction. J Am Soc Echocardiogr. 2012;25(7):733-740.

8. Lorch SM, Ludomirsky A, Singh GK. Maturational and growth-related changes in left ventricular longitudinal strain and strain rate measured by two-dimensional speckle tracking echocardiography in healthy pediatric population. J Am Soc Echocardiogr. 2008;21(11):1207-1215.

9. Ali MT, Yucel E, Bouras S, et al. Myocardial strain is associated with adverse clinical cardiac events in patients treated with anthracyclines. J Am Soc Echocardiogr. 2016;29(6):pp. 522-7 e3.

10. Negishi K, Negishi T, Hare JL, Haluska BA, Plana JC, Marwick $\mathrm{TH}$. Independent and incremental value of deformation indices for prediction of trastuzumab-induced cardiotoxicity. J Am Soc Echocardiogr. 2013;26(5):493-498.

11. Assuncao B, Handschumacher MD, Brunner AM, et al. Acute leukemia is associated with cardiac alterations before chemotherapy. $J$ Am Soc Echocardiogr. 2017;30(11):1111-1118.

12. Lang RM, Badano LP, Mor-Avi V, et al. Recommendations for cardiac chamber quantification by echocardiography in adults: an update from the American society of echocardiography and the European association of cardiovascular imaging. J Am Soc Echocardiogr. 2015;28(1):pp. 1-39 e14.

13. Lopez L, Colan SD, Frommelt PC, et al. Recommendations for quantification methods during the performance of a pediatric echocardiogram: a report from the pediatric measurements writing group of the American society of echocardiography pediatric and congenital heart disease council. J Am Soc Echocardiogr. 2010;23(5):pp. 465-95; quiz 576-7.

14. Khoury PR, Mitsnefes M, Daniels SR, Kimball TR. Age-specific reference intervals for indexed left ventricular mass in children. $J \mathrm{Am}$ Soc Echocardiogr. 2009;22(6):709-714.

15. Eidem BW, Sapp BG, Suarez CR, et al. Usefulness of the myocardial performance index for early detection of anthracycline-induced cardiotoxicity in children. Am J Cardiol. 2001;87(9):pp. 1120-2, A9. 
16. Mousavi N, Tan TC, Ali M, Halpern EF, Wang L, Scherrer-Crosbie M. Echocardiographic parameters of left ventricular size and function as predictors of symptomatic heart failure in patients with a left ventricular ejection fraction of 50-59\% treated with anthracyclines. Eur Heart J Cardiovasc Imaging. 2015;16(9):977-984.

17. Charbonnel C, Convers-Domart R, Rigaudeau S, et al. Assessment of global longitudinal strain at low-dose anthracycline-based chemotherapy, for the prediction of subsequent cardiotoxicity. Eur Heart J Cardiovasc Imaging. 2017;18(4):392-401.

18. Yoon GJ, Telli ML, Kao DP, Matsuda KY, Carlson RW, Witteles RM. Left ventricular dysfunction in patients receiving cardiotoxic cancer therapies are clinicians responding optimally? J Am Coll Cardiol. 2010;56(20):1644-1650.

19. Kalam K, Otahal P, Marwick TH. Prognostic implications of global LV dysfunction: a systematic review and meta-analysis of global longitudinal strain and ejection fraction. Heart. 2014;100(21):1673-1680.

20. Amundsen BH, Helle-Valle T, Edvardsen T, et al. Noninvasive myocardial strain measurement by speckle tracking echocardiography: validation against sonomicrometry and tagged magnetic resonance imaging. J Am Coll Cardiol. 2006;47(4):789-793.

21. Plana JC, Galderisi M, Barac A, et al. Expert consensus for multimodality imaging evaluation of adult patients during and after cancer therapy: a report from the American Society of Echocardiography and the European Association of Cardiovascular Imaging. J Am Soc Echocardiogr. 2014;27(9):911-939.
22. Eidem BW, McMahon CJ, Cohen RR, et al. Impact of cardiac growth on Doppler tissue imaging velocities: a study in healthy children. $J$ Am Soc Echocardiogr. 2004;17(3):212-221.

23. Honda K, Takeshita K, Murotani K, et al. Assessment of left ventricular diastolic function during trastuzumab treatment in patients with HER2-positive breast cancer. Breast Cancer. 2017;24(2):312-318.

24. Harahsheh A, Aggarwal S, Pettersen MD, L'Ecuyer T. Diastolic function in anthracycline-treated children. Cardiol Young. 2015;25(6):1130-1135.

25. Sivasubramanian N, Coker ML, Kurrelmeyer KM, et al. Left ventricular remodeling in transgenic mice with cardiac restricted overexpression of tumor necrosis factor. Circulation. 2001;104(7):826-831.

26. Roberts WC, Bodey GP, Wertlat PT. Wertlake PT. The heart in acute leukemia. A study of 420 autopsy cases. Am J Cardiol. 1968;21(3):388-412.

How to cite this article: Akam-Venkata J, Kadiu G, Galas J, Aggarwal S. Pediatric Malignancies: Is the Prechemotherapy Left Ventricular Function Normal? Echocardiography. 2019;36:1727-1735. https://doi.org/10.1111/echo.14461 